\title{
Question Answering Model in Thai by using Squad Thai Wikipedia dataset
}

\author{
Wicharn Rueangkhajorn \\ School of Information Technology \\ King Mongkut's University of Technology Thonburi \\ Bangkok, Thailand \\ wicharn.keisei@mail.kmutt.ac.th
}

\author{
Assoc.Prof.Dr. Jonathan Hoyin Chan \\ School of Information Technology \\ King Mongkut's University of Technology Thonburi \\ Bangkok, Thailand \\ jonathan@sit.kmutt.ac.th
}

\begin{abstract}
Nowadays, Question Answering is one of the challenge applications in the Natural language processing domain. There are plenty of English language Question Answering model distributed on the model sharing website such as Hugging face hub. Unlike Thai language, there is on a few Thai language Question Answering model distributed on the model sharing website. So, we decided to fine-tune a multilingual Question Answering model to a specify language which is Thai language. The datasets that we will use for training is a Thai Wikipedia dataset from iApp Technology. We have tried to fine-tune on two multilingual model. We also create another dataset to evaluate adaptivity of the model. The result came out to be as satisfy. Both fine-tuned models perform better than base model on evaluation score. We have published Question Answering model to Hugging face hub that will allow people to using these models for others application later.
\end{abstract}

\section{Keywords-Question Answering, NLP, Thai NLP}

\section{INTRODUCTION}

Nowadays, natural language processing is one of the topics in the machine learning field. There are many applications inside natural language processing domain such as chatbot, intent classification, machine translation, sentiment analysis, and many more [2]. One of these applications is called Question Answering. Question Answering application is all about getting the answer from natural language question. Building Question Answering model can be done in two difference approach. The first approach is knowledge base Question Answering. In this approach, the system will try to find an answer likely to be correct to return as an answer. The second approach is textbased Question Answering. In this approach, Question Answering application usually treated as reading comprehension which is finding the answer from the give context. The second approach also require a huge amount of training datasets to produce a good result $[1,3]$.

In Question Answering application, there are many models, which were capable of doing Question Answering, distributed on the model sharing website such as Hugging face hub, but those models are specific to English language. Due to the limited resource of Thai natural language processing, there are only a few models specific to Thai language. That is one of the reasons that we will fine-tune the multilingual model to Thai language. Luckily, there is a public Thai Wikipedia Squad dataset. Therefore, we will this dataset to fine-tune the model and compare the performance with based model that is not fine-tuned specifically to Thai language. In the end, the model will be distributed to model sharing website such as Hugging face hub.
The dataset that was created by human may have some bias which it cannot avoid. This problem can be diagnosed by using test split from another source. This bias is called representation bias. It means a bias of having the train dataset does not represent real world use. In our case, the question in the dataset may not be a natural way human ask the question.

\section{DATASET}

We have used Thai Wikipedia datasets from iApp Technology that were available publicly [4]. This dataset is in the format of The Stanford Question Answering Dataset (SQuAD). The source of this datasets is from Thai Wikipedia website. There are 1,961 Wikipedia document used in this dataset. The context is from only the first section of the article which mean it will be missing some information of this title. This kind of approach also have some advantage in organizing the dataset because the first section of Wikipedia tends to be in the same format unlike other section that may have a bullet point or other format. This dataset already split into train, test, and validation where each part has number of questions like table I.

TABLE I. NUMBER OF QUESTION IN DATASET

\begin{tabular}{|c|c|}
\hline Split & Number of Question \\
\hline Train & 5,829 \\
\hline Test & 723 \\
\hline Validation & 690 \\
\hline Total & 7,242 \\
\hline
\end{tabular}

Several characteristics of this dataset such as context length, answer length, context token and answer token will descript in the table II.

TABLE II. CHARACTERISTIC OF THE DATASET

\begin{tabular}{|c|c|c|c|c|}
\hline \multirow{2}{*}{ Split } & \multicolumn{2}{|c|}{ Length* (mean) } & \multicolumn{2}{c|}{$\begin{array}{c}\text { Number of tokens** } \\
\text { (mean) }\end{array}$} \\
\cline { 2 - 5 } & Context & Answer & Context & Answer \\
\hline Train & 924.55 & 22.87 & 280.19 & 9.98 \\
\hline Test & 880.07 & 22.44 & 268.07 & 9.95 \\
\hline Validation & 829.37 & 22.60 & 250.22 & 9.84 \\
\hline
\end{tabular}

According to table II, the mean length and mean number of tokens of the context seem to have difference in each split of datasets. The mean context length in test is about $95 \%$ of 
train. The mean of context length in validation is about $90 \%$ of train and about $95 \%$ of test. On the other hand, the mean length of answer from all splits seems to be identical to each other. Similarly, the mean number of tokens in test is about $95 \%$ of train. The mean number of tokens in validation is about $90 \%$ of train and about $93 \%$ of test. The mean number of tokens of answer from all splits seem to be very close to each other.

We also create a new test dataset from Wikipedia to evaluate the model. This dataset can help check the adaptivity of the model because it was created by difference people. It may have a representation bias, but this bias is expected to happen in real world for a general domain model. We also use the same approach as the iApp Technology dataset to take only the first section from Thai Wikipedia. We have use cdQA-annotator to help annotate the question and answer. This annotation tool is not maintained by a creator [13]. There are some existing bugs in that tool but it still usable. We can find a easy way to fix those bugs such as refresh the page. The example of annotation tools can be found on Fig. 1.

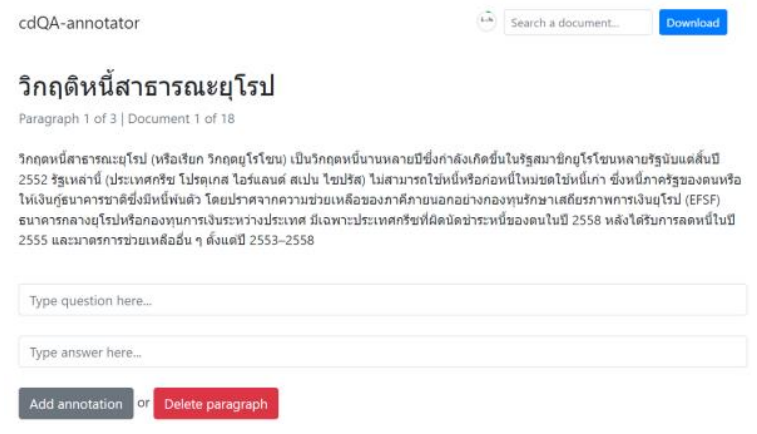

Fig. 1. cdQA-annotator tool

We have annotated 82 rows. The characteristic of our test dataset can be found on table III.

TABLE III. CHARACTERISTIC OF THE DATASET

\begin{tabular}{|c|c|c|c|c|}
\hline \multirow{2}{*}{ Split } & \multicolumn{2}{|c|}{ Length* (mean) } & \multicolumn{2}{c|}{$\begin{array}{c}\text { Number of tokens*** } \\
\text { (mean) }\end{array}$} \\
\cline { 2 - 5 } & Context & Answer & Context & Answer \\
\hline Our Test & 738.62 & 23.85 & 217.81 & 10.36 \\
\hline
\end{tabular}

\section{MODELS}

Since there is a limited resource in Thai natural language processing area, we will use a technique call transfer learning to fine-tune a new model to do Question Answering in Thai. Transfer learning is a process of using a pre-trained language model, that had train with huge datasets, to some specific dataset [3]. For our case, we will use multilingual model distributed on Hugging Face hub as our pre-trained language model. We have done on two type of model which are BERT and RoBERTa to compare a performance.

\section{A. BERT}

BERT stand for Bidirectional Encoder Representations from Transformers which is a pre-train model from an unlabeled text. Since it is bidirectional, BERT will not process a word in sequential. BERT will look at previous or next of the interested word [5].

We will use bert-multi-cased-finetuned-xquadv1 by mrm8488 from Hugging Face hub. This model originated from Google and fine-tuned with Deepmind XQuAD multilingual dataset. This model supports 104 languages [6]. The architecture of this model can be found on table III.

TABLE IV. ARCHITECTURE OF MRM8488/BERT-MULTI-CASEDFINETUNED-XQUADV1 [6]

\begin{tabular}{|c|c|c|c|}
\hline Heads & Layers & Hidden & Params \\
\hline 12 & 12 & 768 & $100 \mathrm{M}$ \\
\hline
\end{tabular}

\section{B. RoBERTa}

RoBERTa stand for Robustly Optimized BERT Pretraining Approach which an improvement of BERT. RoBERTa have train with more datasets BERT which mean it expect to have a better performance than BERT [7].

We will use deepset/xlm-roberta-base-squad2 from Hugging Face hub. This model is based on xlm-roberta-base from Hugging Face. This model supports 100 languages [7, 8]. The architecture of this model can be found on table IV.

TABLE V. ARCHITECTURE OF DEEPSET/XLM-ROBERTA-BASE-SQUAD2 [8]

\begin{tabular}{|c|c|c|c|}
\hline Heads & Layers & Hidden & Params \\
\hline 8 & 12 & 768 & $270 \mathrm{M}$ \\
\hline
\end{tabular}

\section{Hyperparameter}

For both models, we will use same hyperparameter on epoch, learning rate, batch size, and optimizer. Two for epoch. Learning rate is 3e-05. Two for batch size. Adam optimizer with betas from 0.9 to 0.999 and epsilon equal to $1 \mathrm{e}-08$.

\section{EVALUATION}

To verify that the fine-tuned model has better performance than the original model, we will use test split from the Thai Wikipedia datasets. There are two metrics used to evaluation the performance which are F1-score and exact match.

\section{A. F1-score}

F1-score is a common evaluation metric that widely use in machine learning. It also applicable in natural language processing field. It is a good metric if we have equal weight on precision and recall.

$$
F 1=2 * \frac{\text { Precision } * \text { Recall }}{\text { Precision }+ \text { Recall }}
$$

\section{B. Exact Match}

Exact Match F1-score is a common evaluation metric that widely in Question Answering. This metrics is a simple metric. If the prediction matches true answer, then evaluation score will be 1 . If the prediction didn't match true answer, then evaluation score will be 0 .

$$
\begin{gathered}
E M=\frac{\sum_{i=1}^{N} f\left(x_{i}\right)}{N} \\
\text { where } f(x)=\left\{\begin{array}{c}
1, \text { if predicted answer }=\text { true answer } \\
0, \text { otherwise }
\end{array}\right. \\
\text { V. RESULTS }
\end{gathered}
$$

The result of fine-tuned both models came out to be satisfy. We can improve the performance after fine-tuned. RoBERTa base model perform better than BERT base model on the test split of dataset. The evaluation score of both models can be seen on table V. 
TABLE VI. EVALUATION SCORE

\begin{tabular}{|c|l|l|c|c|}
\hline \multirow{2}{*}{ Metrics } & \multicolumn{2}{|c|}{ BERT } & \multicolumn{2}{c|}{ RoBERTa } \\
\cline { 2 - 5 } & $\begin{array}{c}\text { Before fine- } \\
\text { tuned }\end{array}$ & $\begin{array}{c}\text { After fine- } \\
\text { tuned }\end{array}$ & $\begin{array}{c}\text { Before fine- } \\
\text { tuned }\end{array}$ & $\begin{array}{c}\text { After fine- } \\
\text { tuned }\end{array}$ \\
\hline F1-score & 48.26 & 68.10 & 59.99 & 73.62 \\
\hline $\begin{array}{c}\text { Exact } \\
\text { match }\end{array}$ & 31.67 & 57.39 & 44.26 & 62.51 \\
\hline
\end{tabular}

We also evaluate a performance of the trained model using newly create test split and result came out as table VII.

TABLE VII. EVALUATION SCORE FROM OUR TEST SPLIT

\begin{tabular}{|c|c|c|}
\hline Metrics & BERT & RoBERTa \\
\hline F1-score & 59.75 & 75.73 \\
\hline $\begin{array}{c}\text { Exact } \\
\text { match }\end{array}$ & 69.58 & 69.51 \\
\hline
\end{tabular}

We also compare our result with RoBERTa large from deepset[12]. The result came out as table VIII.

TABLE VIII. EVALUATION SCORE FROM ROBERTA LARGE

\begin{tabular}{|c|c|c|}
\hline Metrics & iApp test split & Our test split \\
\hline F1-score & 64.74 & 77.96 \\
\hline $\begin{array}{c}\text { Exact } \\
\text { match }\end{array}$ & 46.61 & 60.97 \\
\hline
\end{tabular}

\section{MODEL DISTRIBUTION}

Since there are only a few Thai Question Answering model publish on the model sharing website, we will publish the model that we already fine-tuned to Hugging Face hub. This can be done by using Hugging Face transformer API [9]. The fine-tuned version of both models can be obtained from Hugging Face hub $[10,11]$. Everyone can use a search feature and search for Question Answering with TH language. There will be the repository of a model and sample usage of it.

\section{CONCLUSION}

Question Answering is one of the most important fields in natural language processing (NLP). There are plenty of resource in English language in Question Answering field. On the other, there are on a few in Thai language. By publishing both models, we hope this will help grow a Thai NLP community further.

\section{APPENDIX}

We have public a model to Hugging Face hub. The model card of the model will consist of a training detail and example of how to use $[10,11]$. The web page also contains an evaluation score like the Fig. 2 and Fig. 3.

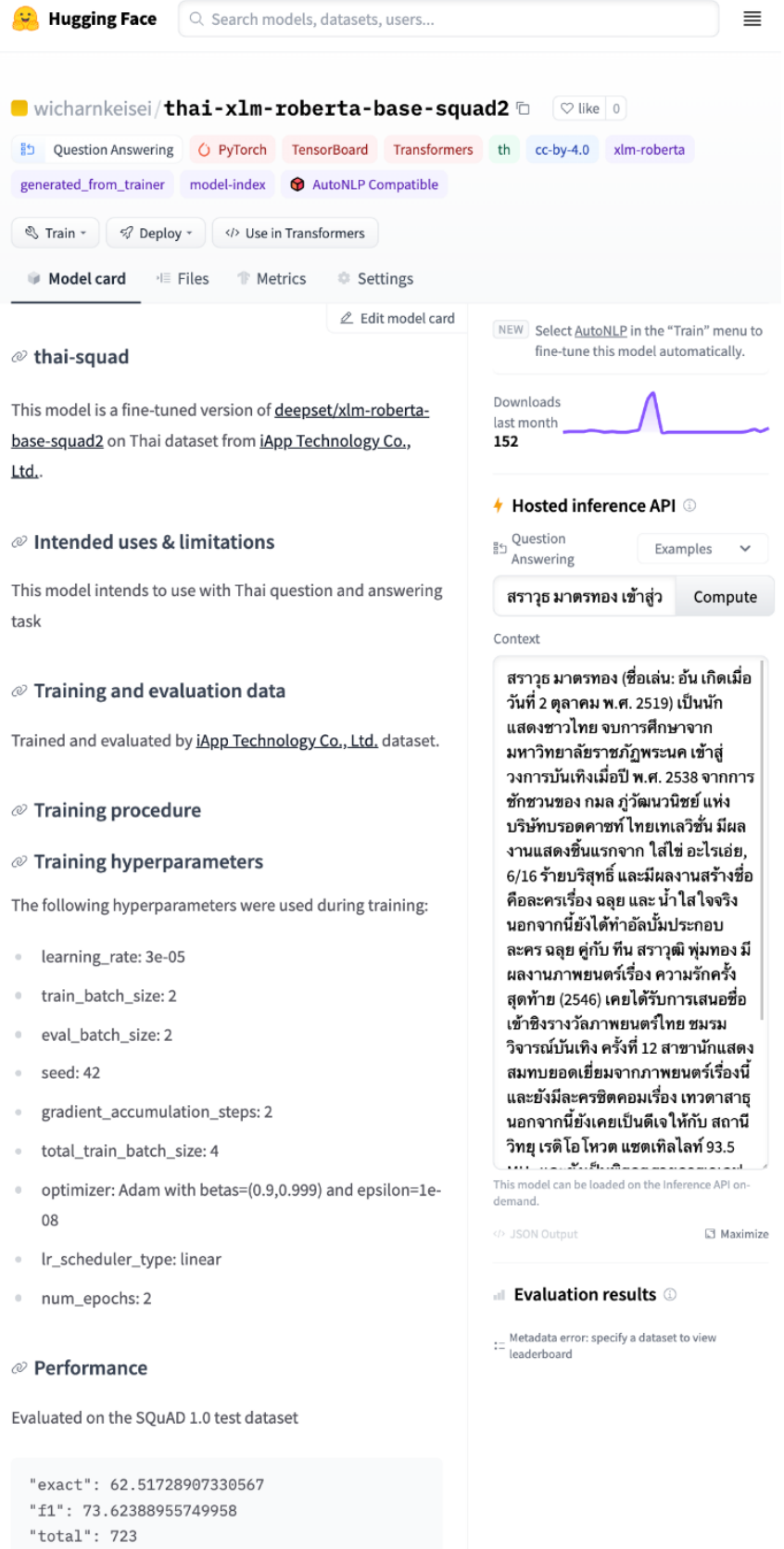

Fig. 2. thai-xlm-roberta-base-squad2 Hugging Face hub page 


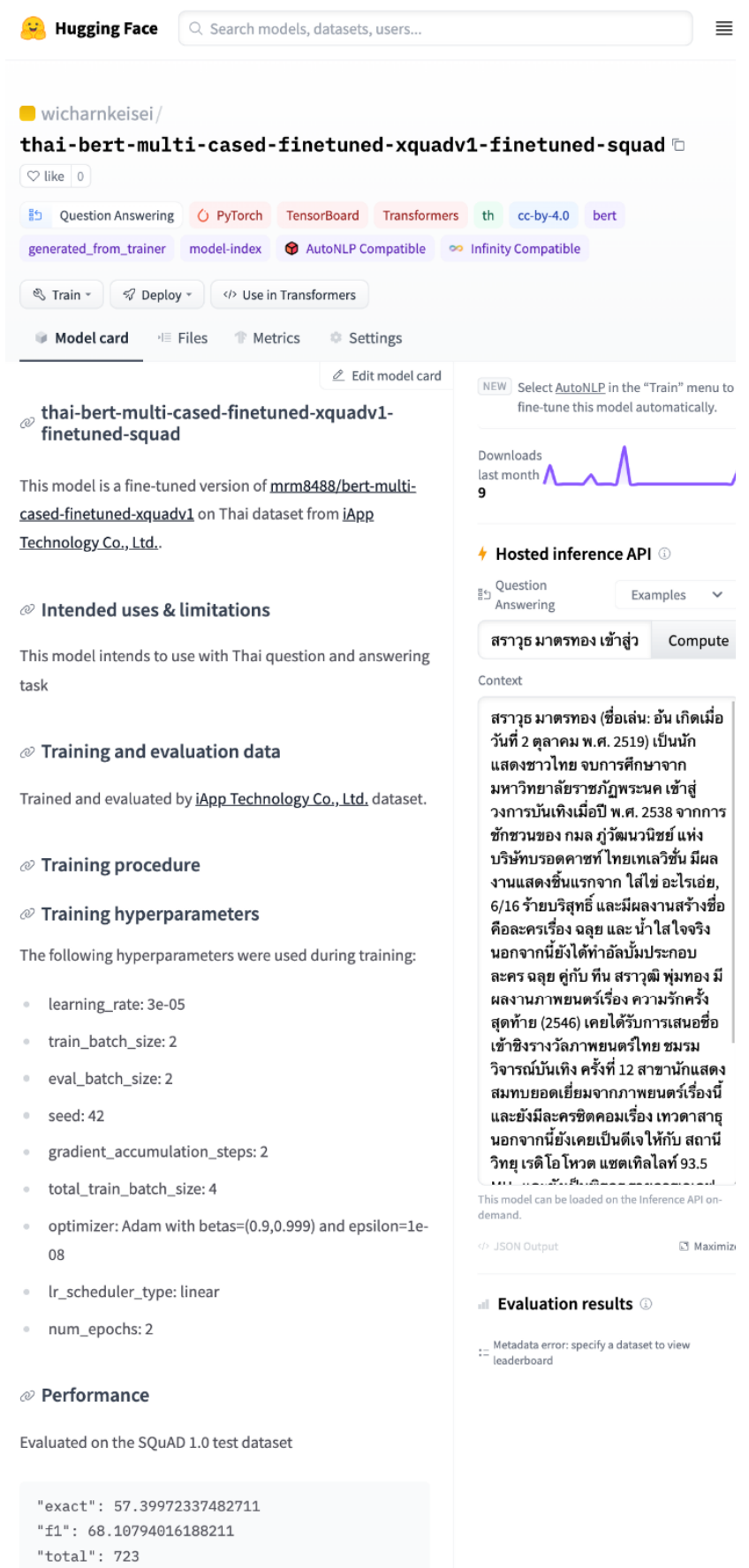

Fig. 3. thai-bert-multi-cased-finetuned-xquadv1-finetuned-squad Hugging Face hub page
[1] P. Rajpurkar, J. Zhang, K. Lopyrev, and P. Liang, "Squad: 100,000+ questions for machine comprehension of text," arXiv.org, 11-Oct2016. [Online]. Available: https://arxiv.org/abs/1606.05250. [Accessed: 12-Nov-2021].

[2] "11 NLP applications \& examples in business," MonkeyLearn Blog, 20-May-2020. [Online]. Available: https://monkeylearn.com/blog/natural-language-processingapplications/. [Accessed: 11-Nov-2021].

[3] K. Alsubhi, A. Jamal, and A. Alhothali, "Pre-trained transformer-based approach for Arabic question answering : A comparative study," arXiv.org, 10-Nov-2021. [Online]. Available: https://arxiv.org/abs/2111.05671. [Accessed: 16-Nov-2021].

[4] Iapp-Technology, "IAPP-Technology/IAPP-wiki-qa-dataset: Open Thai Wikipedia QA dataset made by IAPP technology," GitHub. [Online]. Available: https://github.com/iapp-technology/iapp-wiki-qadataset. [Accessed: 17-Nov-2021].

[5] M. Romero, "MRM8488/Bert-multi-cased-finetuned-xquadv1 . hugging face," mrm8488/bert-multi-cased-finetuned-xquadv1. Hugging Face. [Online]. Available: https://huggingface.co/mrm8488/bert-multi-cased-finetuned-xquadv1. [Accessed: 20-Nov-2021].

[6] M. Artetxe, S. Ruder, and D. Yogatama, "On the cross-lingual transferability of monolingual representations," arXiv.org, 26-May2020. [Online]. Available: https://arxiv.org/abs/1910.11856. [Accessed: 20-Nov-2021].

[7] Y. Liu, M. Ott, N. Goyal, J. Du, M. Joshi, D. Chen, O. Levy, M. Lewis, L. Zettlemoyer, and V. Stoyanov, "Roberta: A robustly optimized Bert pretraining approach," arXiv.org, 26-Jul-2019. [Online]. Available: https://arxiv.org/abs/1907.11692. [Accessed: 20-Nov-2021].

[8] "Pretrained models," Pretrained models - transformers 4.12 .5 documentation. [Online]. Available: https://huggingface.co/transformers/pretrained_models.html. [Accessed: 20-Nov-2021].

[9] "Model sharing and uploading," Model sharing and uploading transformers 4.12.5 documentation. [Online]. Available: https://huggingface.co/transformers/model_sharing.html. [Accessed: 21-Nov-2021].

[10] W. Rueangkhajorn, "wicharnkeisei/thai-bert-multi-cased-finetunedxquadv1-finetuned-squad · hugging face," wicharnkeisei/thai-bertmulti-cased-finetuned-xquadv1-finetuned-squad . Hugging Face. [Online]. Available: https://huggingface.co/wicharnkeisei/thai-bertmulti-cased-finetuned-xquadv1-finetuned-squad. [Accessed: 21-Nov2021].

[11] W. Rueangkhajorn, "Wicharnkeisei/thai-xlm-roberta-base-squad2 . hugging face," wicharnkeisei/thai-xlm-roberta-base-squad $\cdot$ Hugging Face. [Online]. Available: https://huggingface.co/wicharnkeisei/thaixlm-roberta-base-squad2. [Accessed: 21-Nov-2021].

[12] "Deepset/xlm-roberta-large-squad2 · hugging face," deepset/xlmroberta-large-squad2 · Hugging Face. [Online]. Available: https://huggingface.co/deepset/xlm-roberta-large-squad2. [Accessed: 12-Dec-2021].

[13] Cdqa-Suite, "Cdqa-suite/cdqa-annotator: $\Theta$ [not maintained] a webbased annotator for closed-domain question answering datasets with squad format.," GitHub. [Online]. Available: https://github.com/cdqasuite/cdQA-annotator. [Accessed: 13-Dec-2021].

[14] Alexisbcook, "Identifying bias in ai," Kaggle, 09-Nov-2021. [Online]. Available: https://www.kaggle.com/alexisbcook/identifying-bias-inai. [Accessed: 13-Dec-2021].

\section{REFERENCES}

